\title{
Skeletal evidence for variable patterns of handedness in chimpanzees, human hunter-gatherers, and recent British populations
}

\author{
Jay T. Stock, ${ }^{1}$ Meghan K. Shirley, ${ }^{1}$ Lauren A. Sarringhaus, ${ }^{2}$ Tom G. Davies, ${ }^{1,3}$ \\ and Colin N. Shaw ${ }^{1,4}$ \\ ${ }^{1}$ PAVE Research Group, Department of Archaeology and Anthropology, University of Cambridge, Cambridge, United \\ Kingdom. ${ }^{2}$ The Department of Anthropology, University of Michigan, Ann Arbor, Michigan. ${ }^{3}$ Churchill College, Cambridge, \\ United Kingdom. ${ }^{4}$ McDonald Institute for Archaeological Research, University of Cambridge, Cambridge, United Kingdom
}

Address for correspondence: Jay T. Stock, PAVE Research Group, Department of Archaeology and Anthropology, University of Cambridge, Pembroke Street, Cambridge, UK, CB2 3QG. jts34@cam.ac.uk

Previous studies have shown a strong correspondence between long bone bilateral asymmetry and reported handedness. Here, we compare the pattern of asymmetry in mechanical properties of the humerus and second metacarpal of Pan troglodytes, recent British industrial and medieval populations, and a broad range of human hunter-gatherers, to test whether technological variation corresponds with lateralization in bone function. The results suggest that P. troglodytes are left-lateralized in the morphology of the humerus and right-lateralized in the second metacarpal, while all human populations are predominantly right-biased in the morphology of these bones. Among human populations, the second metacarpals of $63 \%$ of hunter-gatherers show right-hand bias, a frequency similar to that found among chimpanzees. In contrast, the medieval and recent British populations show over $80 \%$ right-lateralization in the second metacarpal. The proportion of individuals displaying right-directional asymmetry is less than the expected $\mathbf{9 0 \%}$ among all human groups. The variation observed suggests that the human pattern of right-biased asymmetry developed in a mosaic manner throughout human history, perhaps in response to technological development.

Keywords: laterality; foragers; technology

A majority of humans display a right upper limb bias or use the right hand instead of the left to complete most unimanual tasks. ${ }^{1-3}$ Handedness is considered unique to Homo sapiens ${ }^{4-8}$ as it has yet to be identified with certainty in any other species, including nonhuman primates. ${ }^{9-11}$ Inferences of long-term behavior and activity patterns (particularly for past peoples) can be investigated through patterns of skeletal asymmetry, ${ }^{12}$ based upon functional adaptation of cortical bone to mechanical loading during life. ${ }^{12-14}$ In this study, humeral and second metacarpal rigidity in paired upper limb skeletal elements is investigated among chimpanzees (Pan troglodytes), recent British populations, and a globally representative range of human hunter-gatherers, to compare frequencies of rightleft bias in diaphyseal strength and to investigate variation in inferred hand dominance among these groups.
A broad designation of right- or left-hand dominance can be used to make a distinction between phenotypes, although in reality attributions of handedness often fail to fit clear categories. ${ }^{15,16}$ Knowledge of variation in historic and geographic population frequencies can inform us about both proximate (genetic and nongenetic) and ultimate (evolutionary) factors underlying handedness. ${ }^{17} \mathrm{Ev}$ idence of variation and genetic heritability indicates that selection may be driving the maintenance of the handedness polymorphism in human populations, ${ }^{18-21}$ although at present the question of whether handedness is adaptive or a secondary consequence of brain lateralization remains unresolved. Cultural and other environmental influences also have important selective implications. External (nongenetic) factors may influence the strength and direction of selective forces acting on handedness phenotypes, thus influencing genetic variability 
and corresponding phenotype frequencies in populations. A comprehensive understanding of variation in the prevalence of handedness phenotypes, both temporally and geographically, is essential for testing evolutionary hypotheses. ${ }^{22,23}$

While human right-handedness is often seen as ubiquitous, it does not appear to be fixed in time and space. McManus ${ }^{24}$ suggests that the proportion of left-handers in Western society was around $10 \%$ at the end of the 18 th century, only to decrease during the 19th century and then rise throughout the first half of the 20th century. ${ }^{25,26}$ In 1871, only $4.25 \%$ of Londoners reported that they were lefthanded, ${ }^{24}$ less than half of the $10 \%$ commonly observed in more recent studies in Europe and North America. ${ }^{27-30}$ In a study of more than one million Americans, left-dominance rose from about 3\% in 1910 to about $10 \%$ after World War II. ${ }^{31}$ Similarly, the frequency of left-handed Australians and New Zealanders increased from $2 \%$ in the 19 th century to $13.2 \%$ in the years leading up to $1970 .^{32}$ While these studies show that the frequency of left-handed individuals is somewhat variable, the proportions in western societies over the past two centuries have remained relatively low, rarely exceeding $10 \% .{ }^{33,34}$ There is, however, evidence for a recent trend of increase in the reported frequency of left-handedness among Western populations on account of the relaxation of earlier social stigma. ${ }^{23}$ However, survey data from non-Western countries have highlighted more variable frequencies ranging from 2.5 to $26.9 \%,{ }^{1,3,25,34-37}$ which are undoubtedly influenced by cultural variation in how handedness is self-identified and methodological differences between studies.

Although studies of handedness frequency are difficult to conduct among traditional societies, data from these groups are essential for constructing a detailed picture of the geography of human laterality. ${ }^{2,38}$ From an evolutionary perspective, it is arguably important to examine the handedness polymorphism in traditional cultures, as it may more closely resemble the phenotypic prevalence in ancestral hominin groups. As many of these populations do not engage in Western educational practices, these data can be used to explore hypotheses that Western cultural and technological factors (e.g., schooling) affect handedness. ${ }^{38,39}$ Faurie et al. ${ }^{38}$ reported a range of $3.3-26.9 \%$ across four traditional societies, while a left-hander frequency of $0 \%$ among the Tucano tribe (Colombia) may be attributed to external influence, as the authors reported distinct cultural pressure to use the right hand in this group. ${ }^{40}$ There is considerable evidence that frequencies of handedness are variable between populations and through time, depending upon technology and social factors, despite a universal trend toward right-handedness. Although researchers differ in the degree to which they attribute handedness to culture and genetics, it is widely accepted that both external and internal influences shape the trait phenotypes. ${ }^{4-43}$ Phenotypes (i.e., right and left) may be adaptive or nonadaptive, and natural selection acts on the resultant variation that exists between individuals. ${ }^{44}$

It remains difficult to separate genetic and environmental influences on handedness in humans, ${ }^{43}$ but Laland et al. ${ }^{42}$ have proposed that a gene-culture model (rather than genes or culture alone) could explain right-hand dominance among modern human societies. Socio-cultural factors that have been proposed to influence handedness include social stigma associated with being left-handed, the need to adapt to a world filled with implements designed for righthanders, and the formality of a society's education system. ${ }^{24,42,45}$ It is possible that an underlying genetic basis for handedness may be amplified and driven to a particular side by such socio-cultural factors throughout the life of an individual. ${ }^{46}$

The role of technology in driving lateralization may have a deep evolutionary origin. ${ }^{47-49}$ Frost $^{50}$ and Kimura ${ }^{51}$ have suggested that habitual manual lateralization arose as an evolutionary consequence of behavioral lateralization associated with tool making and use (but see Westergaard and Suomi ${ }^{52}$ ). Observational studies of behavior in both living humans and nonhuman primates have shown that handedness is much stronger for tasks that incorporate tools than for tasks that do not. ${ }^{2,53}$ For example, in a behavioral study G/wi San, Himba and Yanomamö subjects demonstrated weak hand preference for all tasks except those requiring precision grip of a tool. The distinction between tool use and nontool use tasks in these groups serves to highlight the nuanced nature of human handedness, which likely extends from traditional to modernized societies. ${ }^{2}$ The performance of complex and habitual manipulative tasks by humans may be associated with observed differences in handedness between H. sapiens and the rest of the apes. ${ }^{54}$ 
A number of researchers have investigated laterality in great ape genera to investigate whether handedness is a derived condition in the hominin lineage. $7,9,53,55-65$ There has been considerable debate as to whether great apes demonstrate lateralized behavior at the population or species level. ${ }^{53,62,66,67}$ Constraints on handedness imparted by arboreal versus terrestrial activities, ${ }^{7}$ locomotor lateralization $^{68}$ and low- versus high-level tasks ${ }^{59}$ may influence some of the variation in results. Although tool use and manipulatory tasks evoke strong lateralization relative to other types of activity, ${ }^{69,70}$ McGrew and Marchant ${ }^{9}$ have found that this behavior is not consistently manifested beyond the level of the individual in ape populations. In contrast, Hopkins et al. ${ }^{65,71}$ have reported modest (2:1 vs. 9:1 right-handed ratios among modern humans) population-level right bias in chimpanzees, gorillas, and bonobos and left bias in orangutans, which they interpret as evidence that great apes exhibit population level hand preference that differs from humans in degree rather than kind. At present this evidence is based upon the study of captive apes. Future research using different approaches may provide further evidence for population level laterality: the most conservative interpretation at present is that that other great apes do not exhibit consistent, population level handedness. ${ }^{7,55,72}$

Methodological problems may lie at the center of some of the variation in handedness frequencies reported for different populations and between and within species. Cultural variation in perceptions of handedness may influence some of these results. However, inconsistencies in observation or measurement techniques may be a more significant issue. In particular, some studies specifically investigate handedness during fine motor or gross motor tasks, while others either define tasks differently or fail to specify conditions with sufficient clarity to replicate the work. In this light, it is difficult to interpret the collective evidence for variation in handedness among human populations or between species. Here, we use a commonly employed biomechanical method to measure lateralization among chimpanzees, human hunter-gatherers, and recent British populations to investigate broad patterns of variation using the same method of data collection and the same methodological assumptions.

\section{Bone mechanics and the quantification of handedness}

Long-bone diaphyses are the most mechanically sensitive region of the skeleton, and the most reflective of habitual behavior and lateralization ${ }^{44,73,74}$ due to the functional adaptation of cortical bone. ${ }^{12}$ Based on a beam model, the mechanical competence of a bone is estimated via quantification of diaphyseal cross-sectional geometric properties, which are typically measured perpendicular to the long axis of the bone (beam). ${ }^{75}$ Using geometric methods, many studies have demonstrated a clear signal of mechanical adaptation throughout the long bone diaphysis. ${ }^{76-85}$ Diaphyseal responsiveness to mechanical stress via change in shape and size is well supported by evidence from laboratory and clinical research, observations of mechanically restrictive bilateral bone pathologies and studies of modern human athletes. ${ }^{13,14,86-90}$ An individual's mechanical environment during life (loading behavior) can therefore be inferred from the geometry of skeletal elements, ${ }^{75,76,81,91-94}$ and can be evident in the skeleton even many years after an individual has ceased to repetitively engage in a particular activity. ${ }^{95}$

Osteometric studies of bilateral asymmetry using long bone cross-sectional geometry ${ }^{29}$ have demonstrated greater robusticity in an individual's dominant upper limb, ${ }^{90}$ and have been used to investigate handedness among human athletes ${ }^{87,90,91,96,97}$ and the general public, ${ }^{90,98}$ fossil and archaeological specimens, $5,29,73,87,88,99-101$ and nonhuman primates. ${ }^{10,102}$ In terms of the proportion of individuals who show right side dominant directional asymmetry in long bone diaphyses, a study of 992 (924, or $93 \%$, were right-handed) adults from the Baltimore Growth study documented a strong correspondence between second metacarpal cortical thicknesses and self-reported handedness. ${ }^{98}$ Auerbach and Ruff ${ }^{73}$ reported the highest levels of directional asymmetry in humeral diaphysis average diameters, with an average of $76 \%$ showing greater right dimensions than left (79\% among men, 70\% among women), and higher absolute asymmetry among preindustrial groups. While percent directional asymmetry varies considerably between the groups that constitute the sample, the average values are considerably lower than the (about 90\%) right-handed proportions identified from 
the Baltimore Growth Study data. This suggests that either (1) there are differences in the norm of reaction of bone to mechanical loading at different locations in the skeleton; or (2) there is considerable variation in the proportion of handedness among human populations. Given the variation in levels of handedness noted in other studies of handedness among living people, it is also possible that variation in the self-identification of handedness or other methodological differences between studies have influenced the range of study results. By comparing studies of bone morphology as an indicator of handedness, we can provide at least some level of methodological control. A study of variation in diaphyseal total subperiosteal areas (TA) among P. troglodytes found that $64 \%$ had more robust right metacarpals, while a similar proportion $(65.5 \%)$ had greater robusticity of the left humerus. The authors suggested that postural and manipulatory behaviors, perhaps expressed as a trade-off between support and manipulation, may result in observable morphological variation in the robusticity of skeletal elements within the same limb. ${ }^{10}$ While humans may show a pattern of contralateral variation in diaphysis strength from the upper to lower limb, ${ }^{90}$ and between the clavicle and humerus, ${ }^{103}$ the pattern of contralateral variation in upper limb diaphyseal robusticity among chimpanzees is markedly different than the consistent asymmetry throughout the upper limbs noted among humans. ${ }^{73}$

The trends noted above raise issues about the distinction between power and precision, particularly when skeletal data are used to infer hand dominance. Hand dominance is often attributed to individuals based on preference for writing or other fine motor tasks, yet the performance of a range of activities, including those engendering greater loading, must be considered. Although predictions have been made regarding the relationship between strain magnitude and bone modeling, it remains unclear how loads associated with precision (e.g., writing, fine tool manipulation) and power activities (e.g., weight lifting, throwing, hammering) translate to changes in bone morphology. ${ }^{104,105}$ Additionally, the frequency and repetitiveness of precision and power loads likely contribute to bone modeling in distinct ways. ${ }^{29}$ Both high- and lowmagnitude strains affect bone tissue organization, as does the history of habitual activity during de- velopment and adulthood, ${ }^{4,14,105}$ suggesting that all aspects of a bone's strain history should be considered when interpreting bone adaptation. ${ }^{106}$

Despite some evidence for variation in handedness, reviewed above, a recurring theme across the literature on human handedness is the pervasiveness of the $90 \%$ trend. $^{98,107}$ However, the temporal and technological context of variation in handedness is not sufficiently understood, and we know little about variation in prehistory or among populations with different cultural characteristics. Chimpanzees show an unusual pattern of contralateral biomechanical indicators, which stands in contrast to evidence of a near universal trend toward right bias in upper limb skeletal morphometrics among human populations. ${ }^{73}$ Furthermore, the early study by Roy et al. ${ }^{98}$ suggests that over $90 \%$ right-handedness should be expressed in skeletal bilateral asymmetry. The more recent study by Auerbach and Ruff ${ }^{73}$ reported lower frequencies of right-handed directional asymmetry (76\%) among a number of human societies, but did not address the possible relevance of this trend to understanding handedness or its relationship to cultural and technological factors.

The evidence for variation in the mechanical loading of bones, and possible differences in their norm of reaction to this loading, suggest that there is reason to investigate patterns of bilateral asymmetry throughout the limb and body. A previous study demonstrated a pattern of contralateral dominance in the clavicle and humerus among human populations. ${ }^{103}$ The current study tests the simple null hypothesis that humans show a similar pattern of directional asymmetry, with specific emphasis on the humerus and second metacarpal. These bones may be differently involved in precise motor function and general patterns of loading within the limb, and as a result, may show different patterns of directional asymmetry. While the pattern of directional asymmetry in chimpanzee long bone diaphyses reported by Sarringhaus et al. ${ }^{10}$ provides evidence for some degree of lateralization of functional loading in chimpanzee limbs, we predict that human populations will show a universal pattern of greater upper limb lateralization. Following the general trends of the literature on observed and self-reported handedness, we expect approximately $90 \%$ of humans to show greater diaphyseal strength of the right humerus and second metacarpal, and 
Table 1. Skeletal populations used in this study

\begin{tabular}{|c|c|c|c|c|c|c|c|}
\hline Type & Subsample & Location & $n$ & M & $\mathrm{F}$ & Indet. & Reference \\
\hline Pan troglodytes & Wild shot & & 58 & 22 & 28 & 8 & $\begin{array}{l}\text { Sarringhaus } \\
\text { et al. }{ }^{10}\end{array}$ \\
\hline \multirow[t]{13}{*}{ Hunter-gatherers } & Australian Aborigine & Australia & 14 & 7 & 3 & 4 & This study \\
\hline & Andaman Islanders & India & 23 & 10 & 12 & 1 & $\begin{array}{l}\text { Stock and } \\
\text { Pfeiffer }^{76}\end{array}$ \\
\hline & Archaic, Gr. Lakes & Canada & 6 & 3 & 3 & 0 & Stock $^{82}$ \\
\hline & Iberomarusian & $\begin{array}{l}\text { North Africa } \\
\text { (Morocco, } \\
\text { Algeria) }\end{array}$ & 22 & 7 & 3 & 12 & This study \\
\hline & Inuit & Canada & 29 & 17 & 11 & 1 & This study \\
\hline & Kitoi, Serovo, Glazkovo & $\begin{array}{l}\text { Siberia, Russian } \\
\text { Federation }\end{array}$ & 54 & 35 & 15 & 4 & $\begin{array}{l}\text { Stock et al., } \\
\quad 2010^{110}\end{array}$ \\
\hline & Later Stone Age & Kenya & 2 & 1 & 1 & 0 & This study \\
\hline & $\begin{array}{l}\text { Later Stone Age } \\
\text { (Khoesan) }\end{array}$ & South Africa & 30 & 13 & 17 & 0 & $\begin{array}{l}\text { Stock and } \\
\text { Pfeiffer }^{76}\end{array}$ \\
\hline & Later Stone Age & Tanzania & 4 & 2 & 1 & 1 & This study \\
\hline & Mesolithic & France & 4 & 2 & 2 & 0 & This study \\
\hline & Natufian & Levant/Israel & 16 & 4 & 6 & 6 & $\begin{array}{l}\text { Stock et al., } \\
\quad 2005^{111}\end{array}$ \\
\hline & Tasmanian & Tasmanian & 1 & 1 & 0 & 0 & This study \\
\hline & $\begin{array}{l}\text { Yahgan, Tierra del } \\
\text { Fuego }\end{array}$ & Chile & 20 & 9 & 10 & 1 & Stock $^{82}$ \\
\hline Hunter-gatherer total & Subgroups above & $\begin{array}{l}\text { Pooled global } \\
\text { hunter- } \\
\text { gatherer } \\
\text { totals }\end{array}$ & 225 & 111 & 84 & 30 & \\
\hline Medieval British & $\begin{array}{l}\text { Comet Place, Nonsuch } \\
\text { Palace, St. John the } \\
\text { Evangelist }\end{array}$ & $\begin{array}{l}\text { Cambridgeshire, } \\
\text { Surrery, UK }\end{array}$ & 40 & 16 & 24 & 0 & This study \\
\hline 18th-19th Century British & $\begin{array}{l}\text { Christ Church, } \\
\text { Spitalfields }\end{array}$ & London, UK & 39 & 19 & 20 & 0 & This study \\
\hline
\end{tabular}

for this frequency to be consistent across human populations.

\section{Materials and methods}

We compare variation in upper limb long bone diaphyseal morphology among $P$. troglodytes, using published data from Sarringhaus et al. ${ }^{10}$ to unpublished data representing a broad range of hunter-gatherers and both medieval and recent industrial British populations (Table 1). The hunter-gatherer remains represent a broad range of foraging populations from different parts of the world, dating to the late Pleistocene, Holocene and recent historic periods. While the hunter-gatherers encompass a broad range of technologies, they all share a basic, common subsistence strategy, and can generally be assumed to have had relatively homogenous patterns of activity within populations. Agricultural subsistence and subsequent industrialization produce greater behavioral heterogeneity within populations, but it is unknown whether this would result in greater homogeneity between populations. Here, we wanted to test a broad model of the relationship between technology and handedness, thus we have pooled hunter-gatherer data. The late recent British skeletal populations include remains from three medieval cemeteries that have been pooled for the purposes of these analyses, 


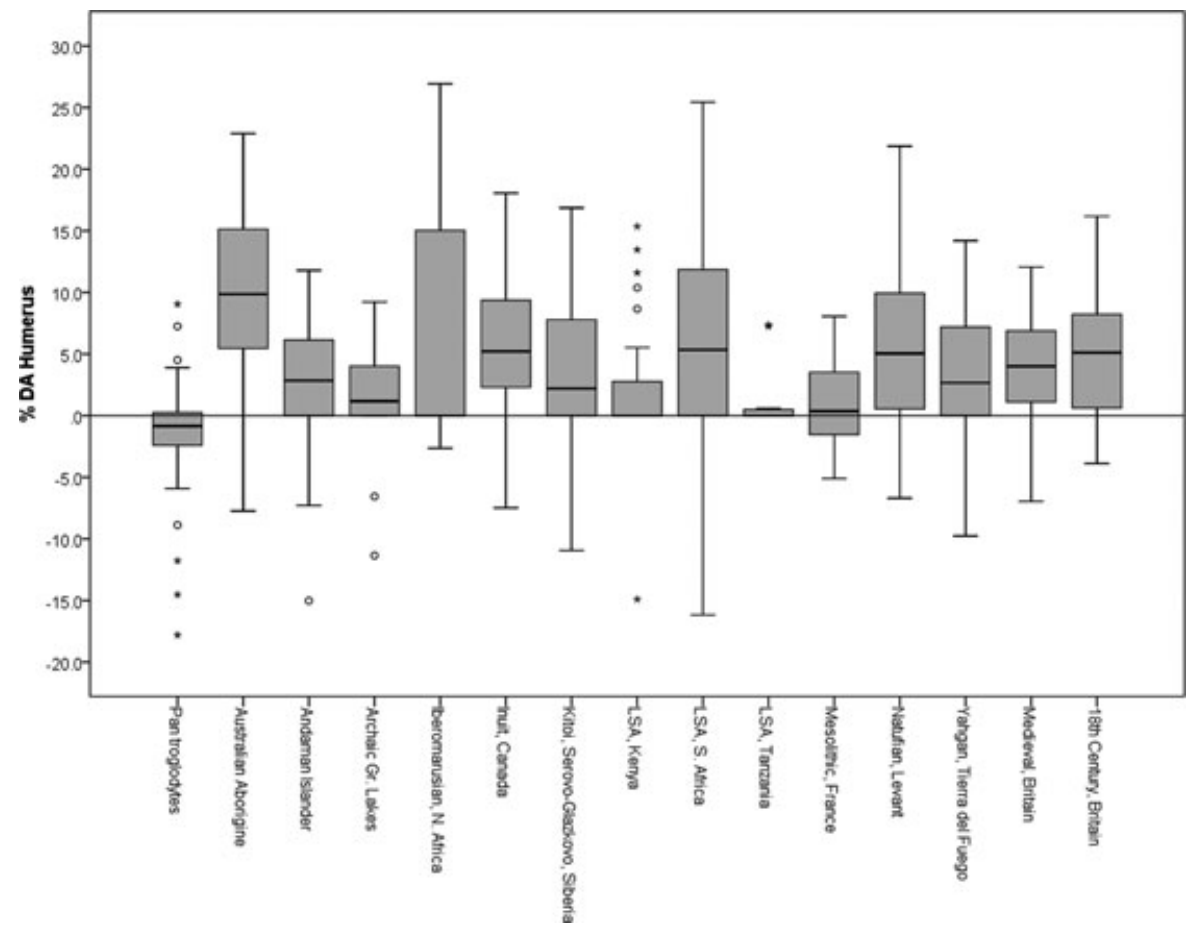

Figure 1. Humeral \% directional asymmetry (\% DA).

as they were not statistically distinguishable, and represented pre-Industrial Revolution populations suspected of having similar activity patterns. The remaining population represents 18th-19th century skeletons from Christ Church, Spitalfields, which dates to the period of the Industrial Revolution. ${ }^{108}$

Cross-sectional dimensions of long bone diaphyses were measured at $50 \%$ of the length of the second metacarpals, and the mid-distal location of humerus $35 \%$ of maximum length among humans, and $40 \%$ among the $P$. troglodytes sample, to take measures at the region of minimal diaphysis circumference and avoid the medial and lateral supracondylar ridges). External diameters were measured to a precision of $0.01 \mathrm{~mm}$ using Mitutoyo digimatic calipers. Total subperiosteal area (TA), quantified from silicone molds taken with Coltene President hydrophilic polysiloxane impression material, was used as a measure of diaphyseal size correlated with strength. ${ }^{83}$ For a small number of cases where TA was not measured, an alternative measurement was used to determine individual directional bias, the product of maximum and minimum diaphyseal breadths (DProd). Values of DProd show a high level of correlation with $\mathrm{TA},{ }^{83}$ and produce comparable percentage directional asymmetry (\% DA) values to those calculated from TA.

Percentage directional asymmetries for each bilaterally paired set of humeri and second metacarpals were calculated using the following equation: $\% \mathrm{DA}=($ right$-\mathrm{left}) /($ average of right and left $) \times 100$.

Positive \% DA values indicate right-side bias, while negative values indicate an asymmetric bias to the left. As the method standardizes for size differences, it allows for comparisons between elements of different dimensions. Differences in \% DA values between $P$. troglodytes, hunter-gatherers, and medieval and recent British groups were tested using Kruskal-Wallis nonparametric tests, as many of the assumptions of ANOVA are violated by such percentage data. Calculations of \% DA from humeral and second metacarpal TA were used to code each individual as either right-biased or left-biased, depending on a positive or negative outcome, respectively. Frequencies of right- and left-biased individuals within each group were then tested for significant deviations from $50 \%$ handedness ratios using $\chi^{2}$ tests. A previous study using this method demonstrated that equivalent results were achieved 


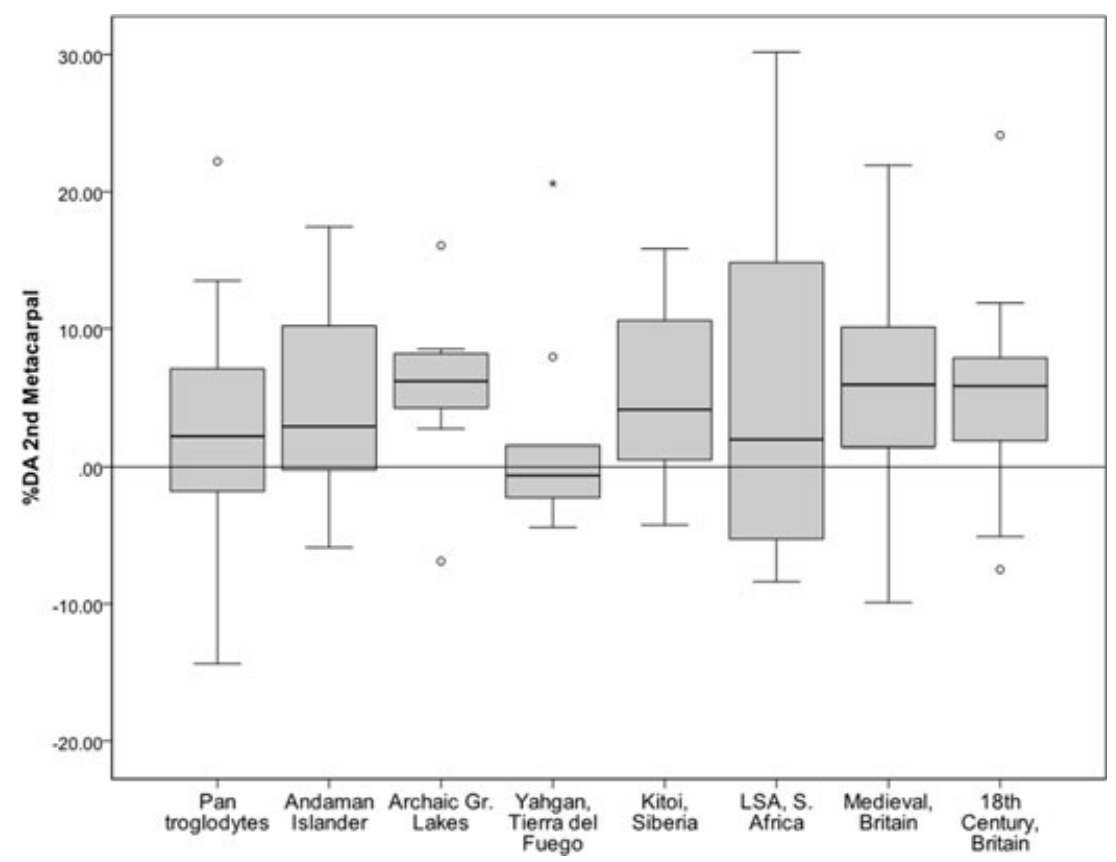

Figure 2. Second metacarpal \% directional asymmetry (\% DA).

when cutoff frequencies of $0 \%, 0.5 \%$ or $1 \%$ DA were used to differentiate handedness from fluctuating asymmetry ${ }^{73}$ which can be defined as random minor deviations from perfect symmetry. ${ }^{109} \mathrm{~A}$ simple $0 \%$ threshold was used in this study to define right- versus left-biased individuals. As there is no a priori reason to expect sexual dimorphism in the mechanical response of bone to bilaterally asymmetric patterns of mechanical loading, sexes were pooled in analyses. ${ }^{98}$ Statistical analyses were undertaken using IBM SPSS Statistics 19.0.

\section{Results}

Percent directional asymmetries of the humeral mid-distal and second metacarpal midshaft locations are presented in Figures 1 and 2. The plots were generated with the hunter-gatherers divided by group to illustrate variation within these populations, however for statistical tests, they were pooled. Mean values of $\%$ DA are presented in Table 2. In the comparison of humeral \% DA, a preponderance of negative values (mean $=-1.22$ ) are found among the $P$. troglodytes series, consistent with the left bias identified previously by Sarringhaus et al. ${ }^{10}$ The medieval and 18th century series had levels of \% DA in the range of $3-5 \%$. While there was considerable variation among the hunter-gatherers, all had pos- itive mean \% DA values, demonstrating a universal trend toward right-biased asymmetry among the human populations. These species/population level differences were statistically significant (KruskalWallis; $\chi^{2}=66.489$; d.f. $=3 ; P \leq 0.001$ ). When we consider $\%$ DA values for the second metacarpal, we note a minor positive (right-biased) trend among $P$. troglodytes, and greater directional right bias among the medieval and recent British series. Again there is considerable variation among huntergatherers, but mean \% DA among the Andaman Islanders, Yahgan, Kitoi, and Later Stone Age South African series are lower than those found among the recent British populations, leading to a slightly lower average percent asymmetry ( 4.43 vs. 5.70 and 5.00). Overall, these population level differences in the magnitude of directional asymmetry were not significant (Kruskal-Wallis; $\chi^{2}=5.672$; d.f. $=3$; $P=0.129$ ).

Relative handedness frequencies are presented in Table 2, as well as the percentage of right-biased individuals within groups and subpopulations. Values are presented for individual hunter-gatherer populations as well as overall means, for which $\chi^{2}$ statistics are presented. Figure 3 presents relative proportions of \% DA in the mid-distal humeri of the $P$. troglodytes, 
Table 2. Mean percent directional asymmetry (\% DA), right and left bias frequencies, and $\chi^{2}$ results

\begin{tabular}{|c|c|c|c|c|c|c|c|c|c|c|c|c|c|}
\hline \multirow[b]{3}{*}{ Type } & \multirow[b]{3}{*}{ Subsample } & \multicolumn{5}{|c|}{ Humerus } & \multirow[b]{3}{*}{$x^{2}$} & \multicolumn{5}{|c|}{ Second metacarpal } & \multirow[b]{3}{*}{$x^{2}$} \\
\hline & & & Mean & & & & & & Mean & & & & \\
\hline & & $n$ & $\% \mathrm{DA}$ & $\mathrm{R}$ & $\mathrm{L}$ & $\%$ Right & & $n$ & $\% \mathrm{DA}^{a}$ & $\mathrm{R}$ & $\mathrm{L}$ & $\%$ Right & \\
\hline Pan troglodytes & & 58 & -1.22 & 20 & 38 & 34.5 & $\begin{array}{c}5.586 \\
(P=0.018)\end{array}$ & 45 & 1.13 & 29 & 16 & 64.4 & $\begin{array}{c}3.756 \\
(P=0.053)\end{array}$ \\
\hline \multirow[t]{13}{*}{ Hunter-gatherers } & Australian Aborigine & 14 & 8.83 & 11 & 3 & 78.5 & & & & & & & \\
\hline & Andaman Islanders & 23 & 3.01 & 20 & 3 & 86.9 & & 19 & 2.56 & 9 & 10 & 47.4 & \\
\hline & Archaic, Gr. Lakes, Canada & 6 & 1.38 & 5 & 1 & 83.3 & & 10 & 2.37 & 7 & 3 & 70.0 & \\
\hline & Iberomarusian, $N$. Africa & 22 & 11.96 & 19 & 3 & 86.3 & & 1 & 15.40 & 1 & 0 & 100 & \\
\hline & Sadlermiut, Canada & 29 & 6.63 & 26 & 3 & 89.7 & & & & & & & \\
\hline & $\begin{array}{l}\text { Kitoi, Serovo, Glazkovo, } \\
\text { Siberia }\end{array}$ & 53 & 3.72 & 40 & 13 & 75.5 & & 19 & 6.27 & 14 & 8 & 73.7 & \\
\hline & LSA, Kenya & & & & & & & 2 & 17.22 & 2 & 0 & 100.0 & \\
\hline & LSA, South Africa & 29 & 7.16 & 25 & 4 & 86.2 & & 18 & 2.83 & 12 & 6 & 66.7 & \\
\hline & LSA, Tanzania & 4 & 3.90 & 4 & 0 & 100.0 & & 3 & -1.27 & 2 & 1 & 66.7 & \\
\hline & Mesolithic, France & 4 & 1.93 & 3 & 1 & 75.0 & & 2 & 4.50 & 1 & 1 & 50.0 & \\
\hline & Natufian, Levant & 16 & 6.81 & 16 & 0 & 100.0 & & 1 & -2.01 & 0 & 1 & 0.0 & \\
\hline & Tasmanian & & & & & & & 1 & -0.41 & 0 & 1 & 0.0 & \\
\hline & Yahgan, Tierra del Fuego & 20 & 2.75 & 15 & 5 & 75.0 & & 4 & 2.47 & 3 & 1 & 75.0 & \\
\hline $\begin{array}{l}\text { Hunter-gatherer } \\
\text { total }\end{array}$ & $\begin{array}{l}\text { Global hunter-gatherer } \\
\text { totals (subgroups above) }\end{array}$ & 220 & 5.70 & 184 & 36 & 83.6 & $\begin{array}{c}113.008 \\
(P \leq 0.001)\end{array}$ & 80 & 4.43 & 50 & 30 & 62.5 & $\begin{array}{c}5.000 \\
(P=0.025)\end{array}$ \\
\hline Medieval British & $\begin{array}{l}\text { Comet Place, Nonsuch Palace, } \\
\text { St. John the Evangelist }\end{array}$ & 40 & 3.85 & 34 & 6 & 85.0 & $\begin{array}{c}19.600 \\
(P \leq 0.001)\end{array}$ & 40 & 5.70 & 33 & 7 & 82.5 & $\begin{array}{c}16.900 \\
(P \leq 0.001)\end{array}$ \\
\hline $\begin{array}{l}\text { 18th-19th Century } \\
\text { British }\end{array}$ & $\begin{array}{l}\text { Christ Church, Spitalfields, } \\
\text { London }\end{array}$ & 39 & 4.78 & 31 & 8 & 79.5 & $\begin{array}{c}13.564 \\
(P \leq 0.001)\end{array}$ & 39 & 5.00 & 33 & 6 & 84.6 & $\begin{array}{c}18.692 \\
(P \leq 0.001)\end{array}$ \\
\hline
\end{tabular}

${ }^{a}$ Hunter-gatherer values calculated from \% DA DProd.

hunter-gatherers, medieval and 18th-19th century British groups. The results demonstrate a strong contrast between $P$. troglodytes, with only $34.5 \%$ right bias in the humeral diaphysis area, when compared to the human groups who average around $80 \%$ right bias. The $\chi^{2}$ tests (Table 2) show that each of these frequencies is significantly different than what would be expected among a nonlateralized population. A comparable plot representing variation in \% DA-determined hand- edness within the second metacarpal is presented in Figure 4. Here all groups show a trend toward rightbiased directional asymmetry. While no population approaches the $90 \%$ that may be predicted based upon recent observational studies, the more recent British groups, representing the medieval and 18 th-19th century periods, both show values above $82 \%$ directional asymmetry. In contrast to this pattern, the $P$. troglodytes and hunter-gatherer groups show second metacarpal \% DA handedness of 64.4

Table 3. Sexual dimorphism in humeral mean percent directional asymmetry (\% DA) within groups

\begin{tabular}{llcccc}
\hline \multicolumn{5}{c}{ Humerus } \\
\hline Type & Subsample & $n$ & Mean \% DA & SD & Kruskal-Wallis \\
\hline Pan troglodytes & Male & 24 & -2.21 & 4.29 & $P=0.325$ \\
Hunter-gatherers & Female & 30 & -0.86 & 1.94 & \\
& Male & 211 & 5.38 & 7.06 & $P \leq 0.001$ \\
Medieval British & Female & 154 & 2.98 & 6.39 & \\
& Male & 24 & 3.90 & 5.12 & $P=0.619$ \\
18th-19th Century British & Female & 16 & 3.78 & 2.86 & \\
& Male & 20 & 4.87 & 5.25 & $P=0.955$ \\
& Female & 19 & 4.68 & 4.81 & \\
\hline
\end{tabular}

${ }^{a}$ Hunter-gatherer values calculated from \% DA DProd. 


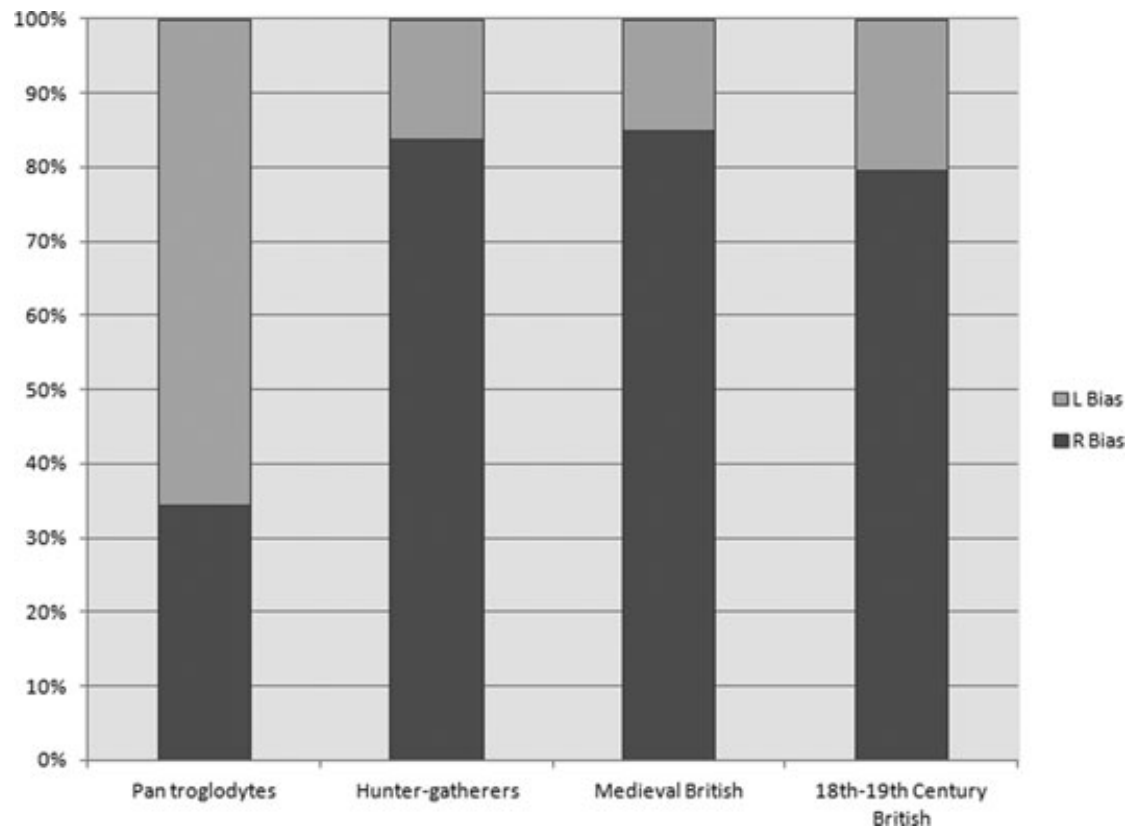

Figure 3. Humeral \% directional asymmetry handedness frequencies.

and $62.5 \%$, respectively. The $\chi^{2}$ tests show that all modern human variation differs significantly from neutral predictions, while the frequency of right-lateralized P. troglodytes is near to significance. The levels of sexual dimorphism in humeral \% DA are higher among chimpanzees and hunter- gatherers than among medieval and 18th century populations (Table 3). The magnitude of sexual dimorphism in average percent directional asymmetry among the medieval or 18th-19th century British groups is nonsignificant and below $0.2 \%$ for both groups. Among chimpanzees, males have, on

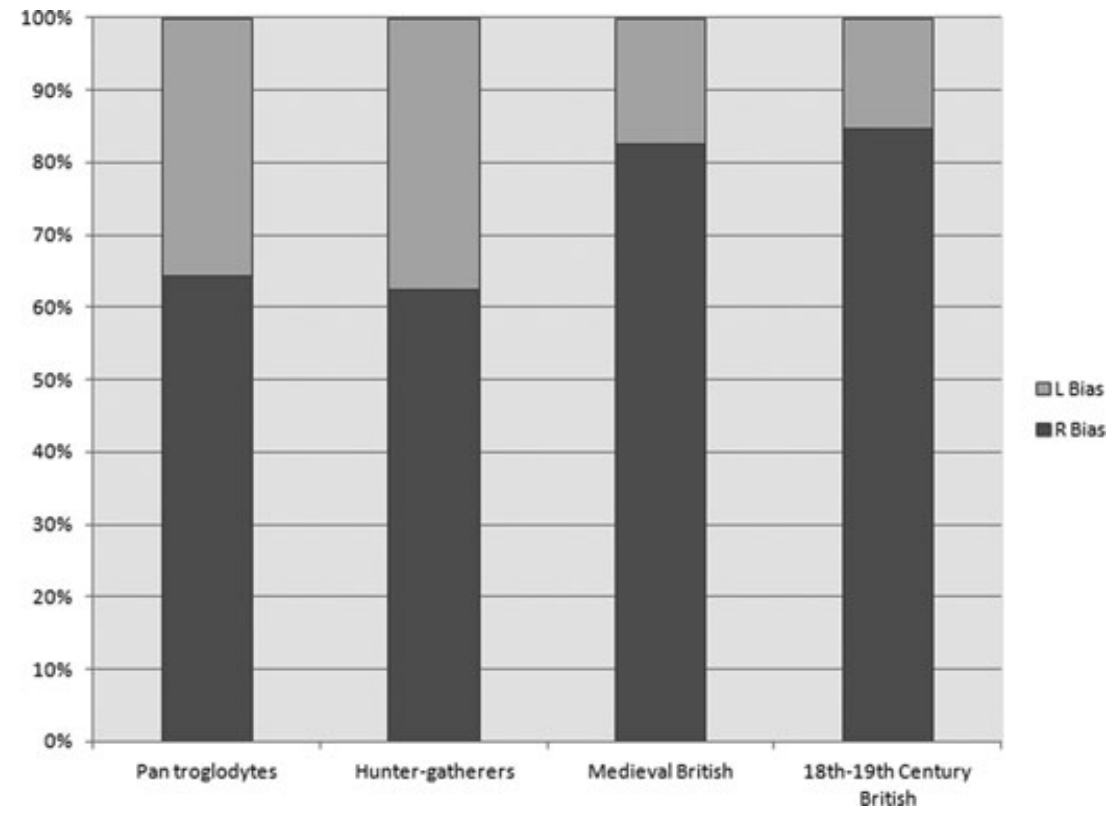

Figure 4. Second metacarpal \% directional asymmetry handedness frequencies. 
average, $1.4 \%$ stronger left humeri than females, but this difference is not significant. Among the huntergatherer groups, males also show greater lateralization, with on average $2.4 \%$ greater strength of the right humerus, a result that is statistically significant on account of the magnitude of the difference and the larger sample size. Unfortunately, similar analyses were not possible for the second metacarpals.

\section{Discussion}

Biomechanical analyses of variation in the bilateral asymmetry of cross-sectional properties of long-bone diaphyses have several advantages over observational studies, including that they provide a means of looking at long-term aggregate mechanical loading rather than short-term preferential hand use for activities that may not elicit a mechanical response. Skeletal analyses also allow for the investigation of laterality in habitual loading of the limbs in the past and among different species. The above analyses highlight interesting trends in the pattern of bilateral asymmetry among chimpanzees and human populations. A previous study has identified a pattern of contralateral variation in upper limb diaphyseal robusticity among chimpanzees, in which there was left bias in humeral rigidity but right bias in second metacarpal rigidity. ${ }^{10}$ Here we demonstrate a pattern of consistent right-limb bias among a range of human populations, which suggests that a consistent trend toward greater frequency of individuals who preferentially load the right limb is a universal human characteristic. This is not surprising given the range of evidence for right dominant lateralization among human populations. Among the medieval and 18th-19th century British populations, the right bias of the humeri and second metacarpals was consistently observed in more than $80 \%$ of individuals, suggesting that among these groups different elements of the limb are similarly and preferentially loaded during lateralized activity. A previous study was suggestive of a strong correlation between second metacarpal cross-sectional geometry and hand preference among living Americans, who showed near $90 \%$ right hand preference. ${ }^{98}$ It is unclear why the percent of right-lateralized individuals sits consistently around $80 \%$ for the humeri of the human groups considered here, but this proportion is similar to those reported for humeral dimensions by Auerbach and Ruff. ${ }^{73}$ It is possible that among a subset of the individuals sampled, the lateralization of mechanical loading is insufficient to engender significant bone remodeling. It is also possible that this difference represents methodological error resulting from variation in the estimation of mechanical properties, leading to different handedness attribution for some individuals who express low asymmetry. It is also possible that the convergence toward $80 \%$ asymmetry in these upper limb elements among different populations represents some true measure of asymmetry in mechanical loading. In this case, variation in reported handedness among living populations may represent sociocultural factors such as social stigma rather than true lateralization of activity or mechanical loading.

One of the most interesting results of the current analysis is the evidence for variation in second metacarpal handedness among human populations. The hunter-gatherers showed much lower levels of right-biased directional asymmetry, with only $62.5 \%$ identified as right-handed on the basis of second metacarpal diaphyseal cross-sectional properties. This value is very similar to that found among the P. troglodytes series at $64.4 \%$. In contrast, both the medieval and 18th-19th century British groups showed values above $80 \%$ rightside bias for second metacarpals, similar to the pattern observed in their humeri and values expected from the literature $(80-90 \%$ right dominance). The pattern of hunter-gatherer variation can be investigated further through the frequencies found among the smaller subsamples of huntergatherers. These population level values illustrate near equal frequencies of right- and left-biased individuals among the Andaman Islanders and low frequencies of right-biased individuals among the Later Stone Age (Khoesan) of South Africa, the midHolocene Kitoi/Serovo/Glazkovo of Siberia, as well as other groups with somewhat smaller sample sizes. This suggests that one population does not drive the trend among foragers, but rather that lower frequencies of right-biased individuals characterize a range of hunter-gatherer groups.

The trend toward right-biased asymmetry in both the humerus and second metacarpal among a range of human populations suggests that this is a widespread trait among humans. The variation observed in the metacarpal in particular suggests that the magnitude of expression of right bias may be variable among human populations and dependent 
upon other factors. A number of studies have documented variation in handedness in recent human history in response to cultural or technological factors. ${ }^{24}$ The fact that similar values are noted in a range of hunter-gatherers suggests that technological factors may be driving the low proportions of right-directional bias in metacarpal morphology. More specifically, more recent proliferation of technologies that promote lateralization of the user (such as some hand tools, industrial machinery, sewing machines, military equipment, musical instruments) may have increased the proportion of right-handed individuals, identifiable through such biomechanical estimates. It also suggests that the human pattern of right-biased asymmetry developed in a mosaic manner throughout human history, and in response to technological development rather than as a simple correlate of brain lateralization.

This paper provides some preliminary evidence for higher levels of sexual dimorphism in bilateral asymmetry among chimpanzees and human hunter-gatherers. The reduced levels of asymmetry noted among the medieval and more recent British samples suggest that some of the long-term trends in bilateral asymmetry may be the result of reduced sexual dimorphism in patterns of habitual mechanical loading associated with technological change. This trend deserves further investigation.

Behaviors and associated skeletal morphology are extremely variable between individuals and within populations, and the relationship between internal (biological) and external (cultural) factors is highly complex. Skeletal tissue is sensitive to various forms of external stimuli, and therefore provides a detailed biological account of an individual's interaction with the environment. ${ }^{109}$ Further research will be required to test the extent of correspondence between handedness and bone morphology among living individuals, to explore whether a frequency of $80 \%$ right-biased individuals truly reflects an $80 \%$ frequency of right-handed individuals. A recent study of this nature has demonstrated a strong correspondence (around 90\%) between humeral robusticity and reported handedness. ${ }^{90}$ This suggests that the frequencies observed in this study may be a reasonable reflection of handedness in past populations and that the similarities and differences observed among the groups considered here are reflective of differences in the lateralization of activity during life. The results of this study should be viewed as preliminary, given the sample sizes of some of the groups studied. Future research should provide a more systematic investigation of variation in human limb asymmetry associated with cultural and behavioral factors. Such work will be required to consider how handedness changed with cultural and subsistence shifts from hunting and gathering to other subsistence systems, and with technological change throughout the Holocene and in recent historic periods.

\section{Acknowledgments}

The authors would like to thank the curators of the skeletal material for access to the collections studied. We would also like to thank the anonymous reviewers for suggestions that have significantly improved this paper.

\section{Conflicts of interest}

The authors declare no conflicts of interest.

\section{References}

1. Raymond, M. \& D. Pontier. 2004. Is there geographical variation in human handedness? Laterality 9: 35-51.

2. Marchant, L.F., W.C. McGrew \& I. Eibl-Eibesfeldt. 1995. Is human handedness universal? Ethological analyses from three traditional cultures. Ethology 101: 239-258.

3. De Agostini, M., A.H. Khamis, A.M. Ahui \& G. Dellatolas. 1997. Environmental influences in hand preference: an African point of view. Brain Cogn. 35: 151-167.

4. Blackburn, A. 2011. Bilateral asymmetry of the humerus during growth and development. Am. J. Phys. Anthropol. 145: 639-646.

5. Cashmore, L.A. 2009. Can hominin handedness be accurately assessed? Ann. Hum. Biol. 36: 624-641.

6. Cashmore, L., N. Uomini \& A. Chapelain. 2008. The evolution of handedness in humans and great apes: a review and current issues. J. Anthropol. Sci. 86: 7-35.

7. Marchant, L.F. \& W.C. McGrew. 2007. Ant fishing by wild chimpanzees is not lateralised. Primates 48: 22-26.

8. Corp, N. \& R.W. Byrne. 2004. Sex difference in chimpanzee handedness. Am. J. Phys. Anthropol. 123: 62-68.

9. McGrew, W.C. \& L.F. Marchant. 2001. Ethological study of manual laterality in the chimpanzees of the Mahale Mountains, Tanzania. Behavior 138: 329-358.

10. Sarringhaus, L.A., J.T. Stock, L.F. Marchant \& W.C. McGrew. 2005. Bilateral asymmetry in the limb bones of the chimpanzee (Pan troglodytes). Am. J. Phys. Anthropol. 128: 840-845.

11. Braccini, S., S. Lambeth, S. Schapiro \& W.T. Fitch. 2010. Bipedal tool use strengthens chimpanzee hand preferences. J. Hum. Evol. 58: 234-241.

12. Ruff, C.B., B. Holt \& E. Trinkaus. 2006. Who's afraid of the big bad Wolff? "Wolffs law" and bone functional adaptation. Am. J. Phys. Anthropol. 129: 484-498. 
13. Shaw, C.N. \& J.T. Stock. 2009a. Intensity, repetitiveness and directionality of habitual adolescent mobility patterns influence the tibial diaphysis morphology in athletes. Am. J. Phys. Anthropol. 140: 149-159.

14. Shaw, C.N. \& J.T. Stock. 2009b. Habitual throwing and swimming correspond with upper limb diaphyseal strength and shape in modern human athletes. Am. J. Phys. Anthropol. 140: 160-172.

15. Llaurens, V., M. Raymond \& C. Faurie. 2009. Why are some people left-handed? An evolutionary perspective. Phil. Trans. R. Soc. B. 364: 881-894.

16. Corballis, M.C. 1997. The genetics and evolution of handedness. Psychol. Rev. 104: 714-727.

17. Schaafsma, S.M. et al. 2012. Handedness in a nonindustrial society challenges the fighting hypothesis as an evolutionary explanation for left-handedness. Evol. Hum. Behav. 33: 94-99.

18. Annett, M. 1985. Left, Right, Hand and Brain: The Right Shift Theory. London: Lawrence Erlbaum Associates, Ltd.

19. McManus, I.C. 1985. Right and left hand skill: failure of the right shift model. Br. J. Psychol. 76: 1-16.

20. Denny, K. \& V. O’Sullivan. 2007. The economic consequences of being left-handed: some sinister results. J. Hum. Resource 42: 353-374.

21. Corballis, M.C. 2009. The evolution and genetics of cerebral asymmetry. Phil. Trans. R. Soc. B. 364: 867-879.

22. Peters, M. 1990. Phenotype in normal left-handers: an understanding of phenotype is the basis for understanding mechanism and inheritance of handedness. In LeftHandedness: Behavioral Implications and Anomalies. 167192. Amsterdam: Elsevier.

23. Steele, J. 2000. Handedness in past human populations: skeletal markers. Laterality 5: 193-220.

24. McManus, I.C. 2009. The history and geography of human handedness. In Language Lateralization and Psychosis. 3757. Cambridge: Cambridge University Press.

25. McManus, I.C. 2002. Right Hand, Left Hand: The Origins of Asymmetry in Brains, Bodies, Atoms and Cultures. London, UK/Cambridge, MA: Weidenfeld and Nicholson/Harvard University Press.

26. McManus, I.C. \& A. Hartigan. 2007. Declining lefthandedness in Victorian England seen in the films of Mitchell and Kenyon. Curr. Biol. 17: R793-R794.

27. Falk, D. 1987. Brain lateralization in primates and its evolution in hominids. Yrbk. Phys. Anthropol. 30: 107-125.

28. Harris, L.J. 1990. Cultural influences on handedness: historical and contemporary evidence. In Left-Handedness: Behavioral Implications and Anomalies. 195-208. Amsterdam: Elsevier.

29. Lazenby, R.A. 2002. Skeletal biology, functional asymmetry and the origins of 'handedness'. J. Theor. Biol. 218: 129-138.

30. Smits, R. 2010. The Puzzle of Left-Handedness. London: Reaktion Books Ltd.

31. Gilbert, A.V. \& C.J. Wysocki. 1992. Hand preference and age in the United States. Neuropsychologia 30: 601-608.

32. Brackenridge, C.J. 1981. Secular variation in handedness over ninety years. Neuropsychologia 19: 459-462.

33. McManus, I.C. 1991. "The inheritance of left-handedness." In Biological Asymmetry and Handedness, Ciba Foundation Symposium 162.251-267. Chichester: John Wiley and Sons.
34. Singh, M., M. Manjary \& G. Dellatolas. 2001. Lateral preferences among Indian schoolchildren. Cortex 37: 231-241.

35. Perelle, I.B. \& L. Ehrman. 1994. An international study of human handedness: the data. Behav. Genet. 24: 217-227.

36. Mandal, M.K., Y. Ida, S. Harizuka \& N. Upadhaya. 1999. Cultural difference in hand preference: evidence from India and Japan. Int. J. Psych. 34: 59-66.

37. Faurie, C. \& M. Raymond. 2005a. Handedness, homicide and negative frequency-dependent selection. Proc. R. Soc. B. 272: 25-28.

38. Faurie, C., W. Schiefenhovel, S. Le Bomin, S. Billiard \& M. Raymond. 2005b. Variation in the frequency of lefthandedness in traditional societies. Curr. Anthropol. 46: 142-147.

39. Geuze, R.H. et al. 2012. Plasticity of lateralization: schooling predicts hand preference but not hand skill asymmetry in a non-industrial society. Neuropsychologia 50: 612-620.

40. Bryden, M.P., A. Ardila \& O. Ardila. 1993. Handedness in native Amazonians. Neuropsychologia 31: 301-308.

41. Ardila A. et al. 1989. Effects of cultural background and education on handedness. Neuropsychologia 27: 893-897.

42. Laland, K.N., J. Kumm, J.D. Van Horn \& M.W. Feldman. 1991. A gene-culture model of human handedness. Behav. Genet. 25: 433-445.

43. Schaafsma, S.M., B.J. Riedstra, K.A. Pfannkuche, A. Bouma \& T.G.G. Groothuis. 2009. Epigenesis of behavioral lateralization in humans and other animals. Phil. Trans. R. Soc. B. 364: 915-927.

44. Buck, L.T., J.T. Stock \& R.A. Foley. 2010. Levels of intraspecific variation within the catarrhine skeleton. Int. J. Primatol. 31: 779-795.

45. Medland, S.E., I.B. Perelle, V. De Monte \& L. Ehrman. 2004. Effects of culture, sex and age on the distribution of handedness: an evaluation of the sensitivity of three measures of handedness. Laterality 9: 287-297.

46. Halpern, M.E., O. Gunturkun, W.D. Hopkins \& L.J. Rogers. 2005. Lateralization of the vertebrate brain: taking the side of model systems. J. Neurosci. 25: 10351-10357.

47. Corballis, M.C. 2003. From mouth to hand: gesture, speech and the evolution of right-handedness. Behav. Brain Sci. 26: 199-260.

48. Westergaard, G.C. 1994. Language, tools and neurobehavioral laterality. Commentary on: Greenfield PM. Language, tools and brain: the ontogeny and phylogeny of hierarchically organized sequential behavior. Behav. Brain Sci. 17: 357-365.

49. Hewes, G.W. 1973. Communication and the gestural origin of language. Curr. Anthropol. 14: 65-84.

50. Frost, G.T. 1980. Tool behavior and the origins of laterality. J. Hum. Evol. 9: 447-459.

51. Kimura, D. 1979. Neuromotor mechanisms in the evolution of human communication. In Neurobiology of Social Communication in Primates. 197-219. San Diego: Academic Press.

52. Westergaard, G.C. \& S.J. Suomi. 1996. Hand preference for stone artefact production and tool-use by monkeys: possible implications for the evolution of right-handedness in hominids. J. Hum. Evol. 30: 291-298.

53. McGrew, W.C. \& L.F. Marchant. 1997. On the other hand: current issues in and meta-analysis of the behavioral 
laterality of limb function in nonhuman primates. Yrbk. Phys. Anthropol. 40: 201-232.

54. Uomini, N.T. 2009. The prehistory of handedness: archaeological data and comparative ethology. J. Hum. Evol. 57: 411-419.

55. Finch, G. 1941. Chimpanzee handedness. Science 94: 117188.

56. Boesch, C. 1991. Handedness in wild chimpanzees. Int. J. Primatol. 12: 541-558.

57. McGrew, W.C. \& L.F. Marchant. 1999. Laterality of hand use pays off in foraging success for wild chimpanzees. Primates 40: 509-513.

58. Byrne, R.W., N. Corp \& J.M.E. Byrne. 2001. Manual dexterity in the gorilla: bimanual and digit role differentiation in a natural task. Anim. Cogn. 4: 347-361.

59. Fagot, J. \& J. Vauclair. 1991. Manual laterality in nonhuman primates: a distinction between handedness and manual specialization. Psychol. Bull. 109: 76-89.

60. Tonooka, R. \& T. Matsuzawa. 1995. Hand preference of captive chimpanzees (Pan troglodytes) in simple reaching for food. Int. J. Primatol. 16: 17-35.

61. Fletcher, A.W. \& J.A. Weghorst. 2005. Laterality of hand function in naturalistically housed chimpanzees (Pan troglodytes). Laterality 10: 219-242.

62. Hopkins, W.D. 2006. Comparative and familial analysis of handedness in great apes. Psychol. Bull. 132: 538-559.

63. Mosquera, M. et al. 2007. Ethological study of manual laterality in naturalistic housed chimpanzees (Pan troglodytes) from the Mona Foundation Sanctuary (Girona, Spain). Laterality 12: 19-30.

64. Llorente, M. et al. 2011. Population-level right-handedness for a coordinated bimanual task in naturalistic housed chimpanzees: replication and extension in 114 animals from Zambia and Spain. Am. J. Primatol. 73: 281-290.

65. Hopkins, W.D. et al. 2011. Hand preferences for coordinated bimanual actions in 777 great apes: implications for the evolution of handedness in hominins. J. Hum. Evol. 60: 605-611.

66. Hopkins, W.D. \& R.D. Morris. 1993. Handedness in great apes: a review of findings. Int. J. Primatol. 14: 1-25.

67. Chapelain, A.S., E. Hogervorst, P. Mbonzo \&W.D. Hopkins. 2011. Hand preferences for bimanual coordination in 77 bonobos (Pan paniscus): Replication and extension. Int. J. Primatol. 32: 491-510.

68. Arcadi, A.C. \& W. Wallauer. 2011. Individual-level lateralization in the asymmetrical gaits of wild chimpanzees ( $P a n$ troglodytes): implications for hand preference and skeletal asymmetry? Behavior 148: 1419-1441.

69. Westergaard, G.C., H.E. Kuhn \& S.J. Suomi. 1998. Laterality of hand function in tufted capuchin monkeys (Cebus apella): comparison between tool use actions and spontaneous non-tool actions. Ethology 104: 119-125.

70. Humle, T. \& T. Matsuzawa. 2009. Laterality in hand use across four tool-use behaviors among the wild chimpanzees of Bossou, Guinea, West Africa. Am. J. Primatol. 71: 40-48.

71. Hopkins, W.D., M.J. Wesley, M.K. Izard, et al. 2004. Chimpanzees (Pan troglodytes) are predominantly right-handed: replication in three populations of apes. Behav. Neurosci. 118: 659-663.
72. Palmer, A.R. 2003. Reply to Hopkins and Cantalupo: Chimpanzee right-handedness reconsidered-sampling issues and data presentation. Am. J. Phys. Anthropol. 121: 382384.

73. Auerbach, B.M. \& C.B. Ruff. 2006. Limb bone bilateral asymmetry: variability and commonality among modern humans. J. Hum. Evol. 50: 203-218.

74. Lieberman, D.E., M.J. Devlin \& O.M. Pearson. 2001. Articular area responses to mechanical loading: effects of exercise, age and skeletal location. Am. J. Phys. Anthropol. 116: 266-277.

75. Ruff, C.B. 2008. "Biomechanical analyses of archaeological human skeletons." In Biological Anthropology of the Human Skeleton. 183-206. Hoboken, NJ: John Wiley and Sons, Inc.

76. Stock, J.T. \& S. Pfeiffer. 2001. Linking structural variability to habitual behaviors: foragers from the southern African Later Stone Age and the Andaman Islands. Am. J. Phys. Anthropol. 115: 337-348.

77. Stock, J.T. \& S.K. Pfeiffer. 2004. Long bone robusticity and subsistence behavior among Later Stone Age foragers of the forest and fynbos biomes of South Africa. J. Archaeol. Sci. 31: 999-1013.

78. Holt, B.M. 2003. Mobility in Upper Palaeolithic and Mesolithic Europe: evidence from the lower limb. Am. J. Phys. Anthropol. 122: 200-215.

79. Lieberman, D.E., O.M. Pearson, J.D. Polk, B. Demes \& A.W. Crompton. 2003. Optimization of bone growth and remodeling in response to loading in tapered mammalian limbs. J. Exper. Biol. 206: 3125-3138.

80. Ruff, C.B. 2005. Mechanical determinants of bone form: insights from skeletal remains. J. Musculoskelet. Neuronal. Interact. 5: 202-212.

81. Carlson, K.J. 2005. Investigating the form-function interface in African apes: relationships between principal moments of area and positional behaviors in femoral and humeral diaphyses. Am. J. Phys. Anthropol. 127: 312-334.

82. Stock, J.T. 2006. Hunter-gatherer postcranial robusticity relative to patterns of mobility, climatic adaptation and selection for tissue economy. Am. J. Phys. Anthropol. 131: 194-204.

83. Stock, J.T. \& C.N. Shaw. 2007. Which measures of diaphyseal robusticity are robust? A comparison of external methods of quantifying the strength of long bone diaphyses to cross-sectional geometric properties. Am. J. Phys. Anthropol. 134: 412-423.

84. Maggiano, I.S. et al. 2008. Cross-sectional analysis of long bones, occupational activities and long-distance trade of the Classic Maya from Xcambó-archaeological and osteological evidence. Am. J. Phys. Anthropol. 136: 470-477.

85. Sparacello V.S. \& O.M. Pearson. 2010. The importance of accounting for the area of the medullary cavity in crosssectional geometry: a test based on the femoral midshaft. Am. J. Phys. Anthropol. 143: 612-624.

86. Biewener, A.A. \& J.E.A. Bertram. 1994. Structural response of growing bone to exercise and disuse. J. Appl. Physiol. 76: 946-955.

87. Trinkaus, E., S.E. Churchill \& C.B. Ruff. 1994. Postcranial robusticity in Homo. II: humeral bilateral asymmetry and bone plasticity. Am. J. Phys. Anthropol. 93: 1-34. 
88. Churchill, S.E. \& V. Formicola. 1997. A case of marked bilateral asymmetry in the upper limbs of an Upper Palaeolithic male from Barma Grande (Liguria), Italy. Int. J. Osteoarchaeol. 7: 18-38.

89. Burr, D.B., A.G. Robling \& C.H. Turner. 2002. Effects of biomechanical stress on bones in animals. Bone 30: 781786.

90. Shaw, C.N. 2011. Is "hand preference" coded in the hominin skeleton? An in-vivo study of bilateral morphological variation. J. Hum. Evol. 61: 480-487.

91. Jones, H.H., J.D. Priest, W.C. Hayes, et al. 1977. Humeral hypertrophy in response to exercise. J. Bone Joint Surg. Am. 59: 204-208.

92. Ruff, C.B. \& J.A. Runestad. 1992. Primate limb bone structural adaptations. Annu. Rev. Anthropol. 21: 407-433.

93. Knüsel, C.J. 2000. Bone adaptation and its relationship to physical activity in the past. In Human Osteology in Archaeology and Forensic Science. 381-401. London: Greenwich Medical Media Ltd.

94. Carlson, K.J., F.E. Grine \& O.M. Pearson. 2007. Robusticity and sexual dimorphism in the postcranium of modern hunter-gatherers from Australia. Am. J. Phys. Anthropol. 134: 9-23.

95. Huddleston, A.L., D. Rockwell, D.N. Kulund \& R.B. Harrison. 1980. Bone mass in lifetime tennis athletes. JAMA 244: 1107-1109.

96. Haapasalo, H., S. Kontulainen, H. Sievanen, et al. 2000. Exercise-induced bone gain is due to enlargement in bone size without a change in volumetric bone density: a peripheral quantitative computed tomography study of the upper arms of male tennis players. Bone 27: 351-357.

97. Daly, R.M., L. Saxon, C.H. Turner, et al. 2004. The relationship between muscle size and bone geometry during growth and in response to exercise. Bone 34: 281287.

98. Roy, T.A., C.B. Ruff \& C.C. Plato. 1994. Hand dominance and bilateral asymmetry in the structure of the second metacarpal. Am. J. Phys. Anthropol. 94: 203-211.

99. Ruff, C.B. \& H.H. Jones. 1981. Bilateral asymmetry in cortical bone of the humerus and tibia: sex and age factors. Hum. Biol. 53: 69-86.
100. Mays, S.A. 2002. Asymmetry in metacarpal cortical bone in a collection of British post-medieval human skeletons. J. Archaeol. Sci. 29: 435-441.

101. Rhodes, J.A. \& C.J. Knüsel. 2005. Activity-related skeletal change in medieval humeri: cross-sectional and architectural alterations. Am. J. Phys. Anthropol. 128: 536546.

102. Carlson, K.J. et al. 2006. Locomotor behavior and long bone morphology in individual free-ranging chimpanzees. J. Hum. Evol. 50: 394-404.

103. Auerbach B.M. \& Raxter M.H. 2008. Patterns of clavicular bilateral asymmetry in relation to the humerus: variation among humans. J. Hum. Evol. 54:663-674.

104. Frost, H.M. 2003. Bone's mechanostat: a 2003 update. Anatom. Record 275A: 1081-1101.

105. Pearson, O.M. \& D.E. Lieberman. 2004. The aging of Wolff's "law": ontogeny and responses to mechanical loading in cortical bone. Yrbk. Phys. Anthropol. 47: 63-99.

106. Fritton, S.P., K.J. McLeod \& C.T. Rubin. 2000. Quantifying the strain history of bone: spatial uniformity and self-similarity of low-magnitude strains. J. Biomech. 33: 317-325.

107. Frayer, D.W. et al. 2012. More than 500,000 years of righthandedness in Europe. Laterality 17: 51-69.

108. Cox, M. 1996. Life and Death in Spitalfields, 1700-1850. York: Council for British Archaeology.

109. Watson P.J. \& Thornhill R. 1994. Fluctuating asymmetry and sexual selection. Trends Ecol. Evol. 9: 21-25.

110. Stock, J.T., V.I. Bazaliiskii, O.I. Goriunova, N.A. Savel'ev \& A.W. Weber. 2010. "Skeletal morphology, climatic adaptation and habitual behaviour among the mid-Holocene populations of the Cis-Baikal, relative to other huntergatherers." In Prehistoric Hunter-Gatherers of the Baikal Region, Siberia: Bioarchaeological Studies of Past Lifeways. A. Weber, M.A. Katzenberg \& T. Schurr, Eds.: 193-216. Philadelphia, PA: University of Pennsylvania Museum of Archaeology and Anthropology Pubs.

111. Stock, J.T., S. Pfeiffer, M. Chazan \& J. Janetski. 2005. The F-81 skeleton from Wadi Mataha, Jordan, and its bearing on human variability in the Epipaleolithic of the Levant. Am. J. Phys. Anthropol. 128: 453-465. 